\title{
A Review of Recent Developments in Low Energy Ion Scattering (LEIS) and Its Applications
}

\author{
Philipp Brüner ${ }^{1}$, Thomas Grehl ${ }^{1}$, Hidde H. Brongersma ${ }^{1}$, Ewald Niehuis ${ }^{1}$ \\ ${ }^{1}$ ION-TOF GmbH, Münster, Germany
}

Low energy ion scattering (LEIS), allows the analysis of a material's surface with a sensitivity of just the topmost atomic layer. In a LEIS experiment, noble gas ions with energies of a few keV are scattered off a sample surface. The energy loss of the ions in the scattering process depends on the mass of the scattering partner, so that the quantitative elemental composition of the sample surface is obtained by recording the energy loss spectrum of the backscattered ions. As ions that are scattered in deeper atomic layers are efficiently neutralized and lose additional energy by nuclear and electronic stopping, the scattering from the first atomic layer can be distinguished from sub-surface scattering [1]. This level of surface sensitivity is unique to LEIS, and provides valuable information for a wide range of sample systems.

With the advent of modern instrumentation in the recent years, namely the introduction of double toroidal analyzers and detectors capable of parallel energy detection (Fig. 1), several orders of magnitude were gained in sensitivity compared to conventional ion scattering equipment. This enables the analysis of delicate real-world samples, for instance a supported catalyst with a low loading of the analyte, whereas the analysis was often restricted to model systems in the past.

One example for such a catalyst system is a diesel exhaust catalyst with a low loading of Pt on the order of $0.1 \%$. As the catalytic action takes place at the surface, an analysis with monolayer sensitivity provides valuable information. The LEIS analysis was applied to a fresh catalyst sample and an artificially aged catalyst sample, showing the change in the elemental surface composition. This gives insight into the appearance and nature of catalyst poisons and the change in the fraction of active catalyst material exposed to the surface.

Similarly, Burriel et al [2] used the monolayer sensitivity of LEIS to study the surface composition of $\mathrm{Sr}$ doped $\mathrm{La}_{2} \mathrm{NiO}_{4}$, a material that is extensively studied for its potential applications as a solid oxide fuel cell (SOFC) cathode. For a SOFC cathode material, the transport properties for ionic oxygen are of crucial importance, and strongly depend on its surface composition in the outer atomic layer. Using LEIS, the total absence of $\mathrm{Ni}$ from the topmost atomic layer of $\mathrm{La}_{2} \mathrm{NiO}_{4}$ was demonstrated, a result that could not be seen with other, less surface sensitive techniques.

In SOFCs with porous electrodes, the active region of the electrolyte/electrode interface is typically inaccessible to surface analytical techniques. For this reason, micro-patterned model systems are employed, which allow studying the active region in a simplified, well-controlled fashion. As a single LEIS measurement collects data from a large sample area on the order of $1 \mathrm{~mm}$, the total spectrum is always the result of integrating over multiple repetitions of the micro-pattern. However, retrospective data analysis allows the separation of the different structures of the micro-pattern by defining "regions of interest". Druce et al [3] studied a model system consisting of $\mathrm{La}_{0.6} \mathrm{Sr}_{0.4} \mathrm{Co}_{0.2} \mathrm{Fe}_{0.8} \mathrm{O}_{3-\mathrm{d}}$ (LSCF) microelectrodes on a single crystal $\mathrm{Zr}_{0.905} \mathrm{Y}_{0.095} \mathrm{O}_{1.9525}$ (YSZ) electrolyte, plus gold strips serving as current collectors. Localized LEIS analysis of the different parts is possible, revealing for instance an 
unexpected signal for La on the YSZ electrode, which might be the result of diffusion, the patterning process or the electrochemical testing procedure.

A wholly different sample system successfully analyzed with LEIS consists of $\mathrm{SnO}_{2}$ nanoparticles with a $\mathrm{SiO}_{2}$ shell for the use in gas sensor applications. The role of the $\mathrm{SiO}_{2}$ shell is the prevention of particle growth. As the $\mathrm{SnO}_{2}$ core must still be accessible, ultra-thin, porous $\mathrm{SiO}_{2}$ layers are desired to act as a spacer between the nanoparticles while still allowing electrical contact [4]. With LEIS, the exact point of layer closure with increasing $\mathrm{SiO}_{2}$ thickness can be reliably detected by the absence of $\mathrm{ZnO}_{2}$ in the outermost atomic layer of the nanoparticles (Fig. 2). Other, less surface sensitive techniques with an information depth of more than one monolayer will detect some $\mathrm{Sn}$ signal, even with a closed $\mathrm{SiO}_{2}$ shell.

References:

[1] H. H. Brongersma et al, Surface Science Reports 62 (2007), p. 63.

[2] M. Burriel et al, Energy Environ. Sci. 7 (2014), p. 311.

[3] J. Druce et al, Nucl. Instr. Meth. B 332 (2014) p. 261.

[4] M. Fuchs et al, Surf. Interface. Anal 42 (2010), p. 1131.

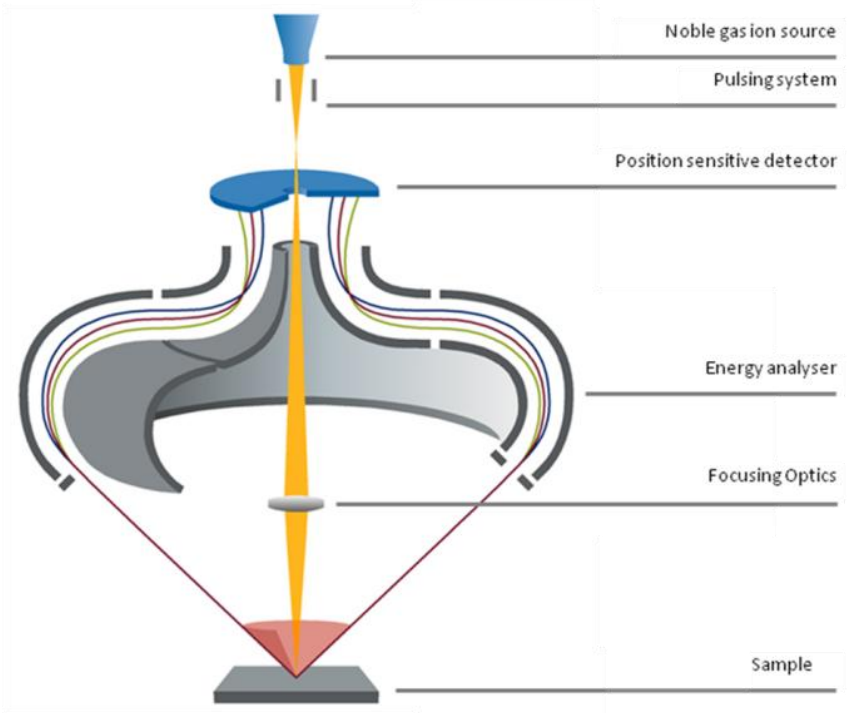

Figure 1. Schematic of a double toroidal energy analyzer used in modern LEIS instruments


Figure 2. SEM image of a core-shell $\mathrm{SnO}_{2} / \mathrm{SiO}_{2}$ nanoparticle thin film (left) and LEIS spectra of nanoparticles (right) with a closed shell showing no $\mathrm{SnO}_{2}$ at the surface, and a porous shell with the $\mathrm{SnO}_{2}$ core exposed to the surface. Samples and SEM image kindly provided by M. Bruns, Karlsruhe Institute of Technology. For further details see [4]. 